\title{
Review
}

\section{Impact of Urinary Incontinence on Women's Sexuality}

\author{
Sílvia Escura Sancho ${ }^{1,2, \dagger}$, Laura Ribera-Torres ${ }^{1,2, \dagger}$, Camil Castelo-Branco ${ }^{1,2,3}$, \\ Sònia Anglès-Acedo ${ }^{1,2, *}$
}

\author{
${ }^{1}$ Pelvic Floor Unit, Working Group of Clinical Sexology, ICGON, Hospital Clínic de Barcelona, University of Barcelona, 08036 Barcelona, Spain \\ ${ }^{2}$ Surgical and Medicosurgery Specialties Departament. Universitat de Barcelona, 08007 Barcelona, Spain \\ ${ }^{3}$ Institut d'Investigacions Biomèdiques August Pi I Sunyer (IDIBAPS), 08036 Barcelona, Spain \\ *Correspondence: sangles@clinic.cat (Sònia Anglès-Acedo) \\ $\dagger$ These authors contributed equally.
}

Academic Editors: Camil Castelo-Branco and Ayse Seyhan

Submitted: 6 December 2021 Revised: 22 December 2021 Accepted: 30 December 2021 Published: 14 February 2022

\begin{abstract}
Objective: Globally, the prevalence of urinary incontinence (UI) in women is estimated to be in the range of $25 \%$ to $45 \%$. Different types of UI exist, being the most frequent ones stress UI, urgency UI and mixed UI. Being affected by UI is associated with a higher risk of sexual inactivity, regardless of its type. Mechanism: It is estimated that $5-38 \%$ patients with UI are sexually inactive due to UI, whereas $25-38 \%$ sexually active women would restrict their sexual activity due to UI. The prevalence of sexual dysfunction within sexually active women with UI is $23-56 \%$. Findings in Brief: In patients with UI, the most frequent sexual dysfunctions are dyspareunia (44\%), female sexual interest/arousal disorder (34\%) and orgasmic disorder (11\%). Therefore, UI has a negative impact on the sexual function of sexually active women with UI. Coital incontinence is defined as the complaint of involuntary loss of urine during or after intercourse. UI with penetration represents any complaint of involuntary loss of urine with vaginal stimulation (either with a sexual toy, manually or with penetration). Orgasmic UI refers to any complaint of involuntary loss of urine occurring at orgasm, regardless of the sexual behaviour that has triggered it. Due to the high prevalence of coital UI $(50-60 \%)$ in the female population and its strongly negative impact on women's sexuality, patients should be specifically asked for coital UI in the gynecologic visits. Conclusions: UI affects between one in four and one in two women and had a negative impact on quality of life. Not only UI has an impact on female sexuality, but also different treatments which are offered to treat it can play a role. The reduction of sexual problems with pelvic floor muscle training in women with stress UI has been proved, being the rate of sexual activity doubled. A significant improvement of the overactive bladder associated to urgency UI and female sexual function has been described after the pharmacological treatment or botulinum toxin. Finally, significant improvement of the sexual function has also been found after midurethral slings for stress UI, as well as for coital UI.
\end{abstract}

Keywords: Urinary incontinence; Stress urinary incontinence; Urgency urinary incontinence; Coital urinary incontinence; Sexual function; Sexual activity; Female sexuality

\section{Introduction}

According to the International Continence Society, UI is a condition defined by involuntary loss of urine. Even though UI prevalence in women widely varies depending on the country [1], the majority of studies globally report numbers in the range of $25 \%$ to $45 \%$ [2]. Different types of UI exist, being the most frequent ones stress UI, urgency UI and mixed UI. Stress UI is defined by involuntary leakage of urine on effort, physical exertion, sneezing or coughing, with a prevalence in the female population estimated between $10 \%$ and $39 \%$. Urgency UI consists of involuntary loss of urine associated with urgency (complaint of sudden, compelling desire to pass urine which is difficult to defer) and approximately affects $1-7 \%$ women. Mixed UI represents the association between stress UI and urgency UI and its prevalence is $7-25 \%[2,3]$.

Despite the fact that UI is not a life-threatening condition, quality of life is negatively affected by it. A crosssectional study including 556 women assessed the impact of UI on quality of life by means of the following validated questionnaires: Medical Outcomes Study 36-item Short-Form Health Survey (SF-36), International Consultation Incontinence Questionnaire Short-Form (ICIQ-SF), King's Health Questionnaire (KHQ), and Pelvic Organ Prolapse Incontinence Sexual Questionnaire (PISQ-12). This study showed that all types of UI interfere in the score of both the general (SF-36) and specific (KHQ and ICIQ-SF) quality of life questionnaires, being women with mixed UI the most affected in this way by their condition [4]. In this line, a study performed in a total of 391 women who were seeking help for all types of UI in a tertiary hospital demonstrated that even mild urinary leakage significantly reduces the quality of life, being less affected by stress UI than by urgency or mixed UI [5].

Quality of life refers to the individual's perception of joy with their own life events, in relation to their goals, expectations, standards and concerns. Therefore, it is important to consider that UI, with its potential impact on the quality of life, may negatively influence its multiple dimen- 
sions, thus altering the physical, social, emotional and sexual wellbeing of the individual.

\section{Urinary Incontinence Impact on Sexual Activity}

Data from published studies shows sexual activity is affected in all the types of UI [6]. All types of UI are associated with a higher risk of sexual inactivity. A case-control study [7] in women from 30 to 60 years old with stress UI, urgency UI or mixed UI in comparison with women without UI, reported a higher rate of sexual inactivity (including penetration, oral sex and masturbation) within the incontinent group (53 vs 29\%). Furthermore, these authors found that age, civil status and UI were independent predictive factors for sexual inactivity.

It is estimated that $5-38 \%$ patients with UI are sexually inactive due to UI, whereas $25-38 \%$ sexually active women would restrict their sexual activity due to UI [6]. The restriction on the sexual activity tends to be due to the fear of incontinence or to the negative emotions such as shame during intercourse, mostly derived from a weakened self-image and self-reliance by this condition [8]. Other reported causes of sexual inactivity are: dyspareunia, nocturnal enuresis, the need of sleeping in separated beds, coital UI or the absence of a partner [6].

\section{Urinary Incontinence Impact on Sexual Function}

The prevalence of sexual dysfunction within sexually active women with UI is $23-56 \%$ [6]. A correlation between the global quality of life of a woman with UI and the impact of her urinary symptoms on her sexual life has been described by Jha et al. [9], independently of the urodynamic diagnosis (stress incontinence, detrusor overactivity and mixed incontinence).

Salonia et al. [10] conducted a study which included 227 women who complained of UI and/or lower urinary tract symptoms. They were assessed with a comprehensive history that included validated questionnaires, a physical examination, an urodynamic testing and the Female Sexual Function Index (FSFI) for the evaluation of the sexual function [10]. In $46 \%$ of these patients with UI a sexual dysfunction was diagnosed, and the most frequent sexual dysfunctions were dyspareunia (44\%), female sexual interest/arousal disorder (34\%) and orgasmic disorder (11\%) [10]. Patients with UI more frequently have sexual fantasies related with the fear to suffer from an episode of urine leakage during intercourse. Such fear may trigger sexual anxiety, a decrease in the sexual activity, low desire and arousal, dyspareunia and a decrease in the orgasmic frequency $[10,11]$.

Regarding the impact of the UI on the partner relationship, a study including 206 women with UI and/or urgency, which focused on the 99 sexually active heterosexual couples who wanted to participate, showed that $49 \%$ of the women reported concerns about having urinary leakage during sexual activity, whereas most of their male partners (94\%) did not [12].

Therefore, as stated in the systematic review of Pinheiro et al. [13], UI has a negative impact on the sexual function. It is probable that this interference contributes, directly or indirectly, to the avoidance of sexual activity. Nevertheless, the way in which this influence occurs is not yet clear [13].

When comparing the different types of UI, the majority of studies suggest patients with mixed UI more frequently suffer from sexual dysfunctions, followed by patients with urgency UI and patients with stress UI [6]. Focalising on the urgency UI, a case-control study with 135 sexually active women with overactive bladder and detrusor overactivity and 112 healthy women, reported a significantly lower score both in the global sexual function and its dimensions in the case group, using a generic sexual questionnaire (FSFI) [14].

Regarding stress UI, qualitative studies evaluate female's sexuality affectation secondary to an altered body image. A study published by Roos [15] reported a $60 \%$ rate of negative autoevaluation of sexual life within sexually active women with partner waiting for an antiincontinence surgery. Women presented with an affected corporal image and an alteration of multiple sexuality dimensions such as motivation, arousal and orgasm. Furthermore, the presence of coital incontinence, or the fear of this condition, was associated with interest, arousal, orgasm and pain disorders.

\section{Urinary Incontinence during Sexual Activity}

Taking the above information into consideration, the evaluation of UI during sexual activity seems an important issue to determine whether this symptom leads to a major impact on sexuality of incontinent women.

Traditionally, the study of UI during sexual activity has been focalised on coital incontinence, defined as the complaint of involuntary loss of urine occurring during or after vaginal intercourse [16]. Coital UI has an estimated prevalence of $50-60 \%$ within incontinent sexually active women, according to studies mainly performed in heterosexual female population $[9,17]$. An epidemiological international multicenter study which included 1041 sexually active women with UI from Italy, Greece, the United States and Egypt, observed that the prevalence of coital UI was $53.8 \%$ [18].

In a cross-sectional multicentric Spanish study conducted in sexually active women who attended the gynecologist seeking treatment for UI and/or overactive bladder, at least one out of three of them had coital UI [11]. Forty per cent of these women reported an either moderate or big impact on their quality of life measured by means of the specific questionnaire King's Health Questionnaire. 
Observing in detail the answers given to the items about impact on couple and sexual life comprised in the personal relations dimension, it is possible to verify that women with coital UI have higher scores than those without it [11]. Furthermore, in the multiple regression model in which age and body mass index were entered along with all the variables of urinary symptoms, the only variable that demonstrated independent association with a worse quality of life was coital UI. For this reason, it was concluded that more research is needed about the presence of this symptom in women with urinary symptoms and its relationship with patientperceived severity and sexual dysfunction [11]. Similar to this results, Illiano et al. [18] found that women with coital UI were more affected by UI on their daily life compared to women without coital UI.

A higher prevalence of coital UI (60\%) was reported in another cross-sectional study conducted in women with UI who subsequently underwent an urodynamic assessment [9]. No difference in the prevalence of coital UI when the different urodynamic diagnosis (stress incontinence, detrusor overactivity and mixed incontinence) were compared, whereas women with normal urodynamic findings were less likely to experience UI during coital activity $(p=0.035)$. The physiopathological mechanism underlying coital UI is still controversial. Classically, this symptom has been divided into that occurring with penetration and that occurring at orgasm. Coital UI occurring with penetration has been associated with urodynamic stress UI and coital UI occurring at orgasm has been associated with urodynamic detrusor overactivity $[6,19]$. Nevertheless, more recent studies have suggested the association between coital UI and urethral sphincter deficiency [18,20,21], as an association between orgasmic UI and urodynamic stress incontinence by intrinsic sphincter deficiency has been observed [18,21]. Concretely, Lau et al. [20] conducted an observational study with 505 sexually active women with UI in which univariate analysis both a higher body mass index and a lower maximal urethral closure pressure $\left(\leq 30 \mathrm{~cm} \mathrm{H}_{2} \mathrm{O}\right)$ were suggested to be possible predictors of coital UI. Moreover, the multivariate analysis showed that the lower maximal urethral closure pressure was an independently significant predictor [20]. However, in contrast to previous publication, Illiano et al. [18] found in their multivariate analysis that women with BMI $>25 \mathrm{~kg} / \mathrm{m}^{2}$ is an independent risk factor for coital UI. Furthermore, El-Azab et al. [21] in a study that included 90 patients with UI, 60 of whom had coital UI, reported a significantly higher prevalence of coital UI among women with stress UI $(89.4 \%)$, in comparison to those with detrusor overactivity $(33.3 \%)$. Other factors that were significantly associated with coital UI were parity, pelvic organ prolapse and stress UI [21]. Therefore, coital UI is a pathological sign caused by either an urethral disorder, detrusor overactivity or a combination of both, for which treatment is required [22].
In accordance with the current terminology of the International Continence Society (ICS)/International Urogynecological Association (IUGA) [16] all women can be evaluated with the new definitions, regardless of their sexual orientation and the existence or not of a partnership. At present, UI with penetration represents any complaint of involuntary loss of urine with vaginal stimulation (either with a sexual toy, manually or with penetration). Orgasmic UI refers to any complaint of involuntary loss of urine occurring at orgasm, regardless of the sexual behaviour that has triggered it.

On the other hand, it is important to stablish an appropriate differential diagnosis between fluids which are expelled during the female sexual response, either during arousal and/or orgasm [22]. Vaginal lubrication is a blood plasma ultrafiltrate of variable amount, representing a transvaginal transudate which increments with sexual stimulation and correlates with the arousal phase of the sexual response. Female ejaculation is another fluid that has been described which occurs during orgasm. It consists of the secretion of a few milliliters of a dense and milky liquid through the urethra, being originated in the paraurethral (Skene's) glands, which are considered to be the female prostate or $\mathrm{G}$ spot for some authors, as it contains prostate specific antigen (PSA) [23]. However, at orgasm, another fluid can be expelled: squirting or gushing. It is defined as an involuntary expulsion of a large quantity of diluted urine during sexual stimulation of the clitoro-urethrovaginal complex. Therefore, both the female ejaculation and squirting are expelled through the urethra, but their origin (paraurethral glands and bladder, respectively) and, as a consequence, composition, is different. They can be distinguished because squirting is a clear and watery liquid while female ejaculation tends to be a milky substance. These three physiological processes can occur either isolatedly or coexist in some women. All of them are considered a positive phenomenon which improves the sexual life of both the woman herself and her sexual partners [22].

Conversely, women with UI with concomitant coital UI negatively experience this specific symptom, which has an influence in different dimensions of the quality of life [24,25] (e.g., emotional, relational and sexual). In relation to this, Illiano et al. [18] showed that coital UI was associated with increased severity of UI according to the ICIQ-SF scores, as well as with higher impact on the quality and frequency of sexual activity. Due to the high prevalence of coital UI in the female population and its strongly negative impact on women's sexuality, patients should be specifically asked for coital UI in the gynecologic visits [17].

\section{Impact of Urinary Incontinence Treatment on Sexuality}

Not only UI has an impact on female sexuality, but also different treatments which are offered to treat it can play a role. Conservative measures (lifestyle modifications 
and pelvic floor muscle training), medicines and surgery are the most frequently used treatments for UI. Other treatments which tend to be used in refractory cases are intravesical injection of botulinum toxin, neuromodulation of the sacral nerve and posterior tibial nerve stimulation.

\subsection{Pelvic Floor Muscle Training and Sexuality}

The female sexual function is inherently complex, as it involves an interplay of multiple physical, hormonal, emotional, contextual and sociocultural factors, among others which have been implicated. Taking the physiology of the sexual response into account, pelvic floor muscles are considered to play an important role in both the female arousal and orgasm [26,27].

Different studies have described an association between pelvic floor muscle function and better rates of sexual activity and sexual function in women with pelvic floor dysfunctions, even though causality has not been stablished due to a lack of randomized studies. In a multicenter study of 585 women with pelvic floor disorders from the United States and the United Kingdom it was observed that women with a strong pelvic floor were more likely to report sexual activity than women with weak strength, but no differences were found between women with normal and hypoactive pelvic floor tone [28]. In this line, the reduction of sexual problems with pelvic floor muscle training in women with stress UI has been proved [28], being the rate of sexual activity doubled.

Furthermore, an improvement of sexual variables is found in women with any type of UI who have performed pelvic floor muscle training, when compared with controls [29]. Probably, this positive impact on sexual life after treatment is due to, not only an improvement in the muscle function, but also an improvement of the UI symptoms, which minimyze its impact on sexuality.

It is also important to note that learning to coordinate pelvic floor muscles (voluntary contraction and relaxation) provides patients with control over a part of their bodies, which empowers them in their sexuality and can frequently constitute the door to work self-efficacy and self-esteem. A reduction in sexual problems of women with UI who perform pelvic floor muscle training has also been suggested by means of self-image improvement [30].

\subsection{Urgency Urinary Incontinence Treatment and Sexuality}

A meta-analysis has evaluated the impact on sexual function of overactive bladder associated to urgency UI, and also of its treatments measured by means of the FSFI questionnaire in the majority of studies [31]. In this systematic review, overactive bladder with UI (overactive bladderwet) was reported to be the most important factor affecting women's sexuality. A significant improvement of the overactive bladder associated to urgency UI and female sexual function, was described after anticholinergic, beta- adrenergic and botulinum toxin therapies. For this reason, authors conclude that women with overactive bladder associated with urgency UI and sexual dysfunction could benefit from a targeted therapy for UI symptoms. Frequently, fear or anxiety of feeling dirty or with bad smell make women with urgency UI incorporate adaptive maneuvers [6], such as urinating before and after partnered sexual activity, which influence how intimacy, proximity and partner dynamics are dealt with.

Possibly, the decrease in the urgency UI episodes with the treatment could also improve these more global aspects of the sexual life. In relation to this, Wang et al. [32] performed a study including 323 women with overactive bladder and demonstrated the effect of the severity of its symptoms on the rate of female sexual dysfunction and partners' sexual satisfaction. When the different domains of the overactive bladder were analyzed, the urgency symptoms showed a significant effect on the female sexual function, as more severity of urgency UI has been associated with a higher rate of female sexual dysfunction [32]. In this way, UI was closely associated with partners' sexual satisfaction [32]. Serati et al. [17] reported that the form of coital UI during orgasm is curable by antimuscarinic treatment in about $60 \%$ of cases when associated with detrusor overactivity.

Therefore, patients need to be informed in detail about the potential benefits in terms of improvement in the sexual function with urgency UI treatment.

\subsection{Stress Urinary Incontinence Surgical Treatment and Sexuality}

A meta-analysis, including 22 studies, in which sexual function is evaluated after the collocation of retropubic and transobturator tension-free midurethral sling for the treatment of stress UI, confirms a significant improvement of the sexual function after stress UI surgical treatments. Results were evaluated in the mid-term (12-24 months after surgery) by means of the global score of the specific questionnaire PISQ-12 and the generic questionnaire FSFI and also by the 6 sexual dimensions of the FSFI questionnaire. A significant decrease by nearly 6-fold in the post-operative coital UI was also observed [33], being only based on 998 patients and 7 studies which assessed coital UI before and after surgery. A recent publication [34] specifically analysed the effect of retropubic tension-free midurethral sling for stress UI on coital UI. It was a prospective observational study conducted in women with urodynamic stress UI and concomitant coital UI. Among the 82 women who answered the post-operative Patient Global Impression-Improvement (PGI-I) scale for coital UI to assess the patient-reported success rate, in the pre-operative clinical assessment 58\% stated they experienced UI during penetration, $15 \%$ during orgasm and $25 \%$ during both situations. After surgery, $86 \%$ reported being very much or much better in the evaluation, being the first study which shows the success rates after an- 
tiincontinence surgery according to each type of coital UI (91\% in women with UI with penetration, 69\% in women with orgasmic UI and $85 \%$ in women with both types of coital UI). In addition, a statistically significant improvement in the FSFI global sexual function score was found after the retropubic tension-free midurethral sling procedure. Therefore, according to these results, most of the women with stress UI and concomitant coital UI will improve their sexuality and, therefore, their quality of life after surgery.

Nevertheless, having performed a surgery in the anterior vaginal wall, anatomic, physiological, vascular, neurological and hormonal alterations can occur. Variations in this anatomical region could alter the vaginal sensitivity or vasocongestion by an affectation of its innervation, thus altering the female sexual function of a minority subgroup of women. Therefore, it is fundamental to evaluate women in the post-operative in order to detect in time possible complications of the antiincontinence techniques in the sexual sphere.

\section{Conclusions}

UI affects between one in four and one in two women, being stress UI the most prevalent type followed by mixed UI and urgency UI. Quality of life is negatively affected by UI, and also the physical, social, emotional and sexual wellbeing of the individual may be altered by it. All types of UI affect the sexual activity rate and the prevalence of sexual dysfunction within sexually active women with UI is high. Moreover, almost half of sexually active women with UI have coital UI. Treatments for UI such as conservative measures, medicines and surgery may have a positive impact on female sexuality.

Most of the research on sexual health in women with UI does not intend to identify the proportion of problems which are directly related to UI. For this reason, the measurement of the real impact of this dysfunction and its treatments on sexual health remains a complicated issue. Good quality investigation on sexual health in women with UI is required and it should be addressed to quantify the aspects of female sexual health which become affected by UI itself. This would lead to an improvement of the management strategies of these patients and the information being provided to them, which could comprise realistic expectations about the likely impact of the specific treatments.

\section{Author Contributions}

SES and SAA designed the research study, performed the research, analyzed the data and wrote the manuscript. LRT and CCB provided advice on manuscript editing. All authors contributed to editorial changes in the manuscript. All authors read and approved the final manuscript.

\section{Ethics Approval and Consent to Participate}

Not applicable.

\section{Acknowledgment}

Thanks to all the peer reviewers for their opinions and suggestions.

\section{Funding}

This research received no external funding.

\section{Conflict of Interest}

The authors declare no conflict of interest. $\mathrm{CCB}$ is serving as one of the Editorial Board members and Guest editors of this journal. We declare that $\mathrm{CCB}$ had no involvement in the peer review of this article and has no access to information regarding its peer review. Full responsibility for the editorial process for this article was delegated to MD.

\section{References}

[1] Mostafaei H, Sadeghi-Bazargani H, Hajebrahimi S, Salehi-Pourmehr H, Ghojazadeh M, Onur R, et al. Prevalence of female urinary incontinence in the developing world: a systematic review and meta-analysis-a Report from the Developing World Committee of the International Continence Society and Iranian Research Center for Evidence Based Medicine. Neurourology and Urodynamics. 2020; 39: 1063-1086.

[2] Milsom I, Altman D, Cartwright R, Lapitan MC, Nelson R, Sillén $\mathrm{U}$, et al. Epidemiology of urinary incontinence (UI) and other lower urinary tract symptoms (LUTS), pelvic organ prolapse (POP) and anal (AI) incontinence. In Paul Abrams, L. Cardozo, S. Khoury \& A.J. Wein (Eds). Incontinence: 5th International Consultation on Incontinence, Paris, February 2012 (5th ed ed., pp. 15-107). ICUD-EAU.

[3] Haylen BT, de Ridder D, Freeman RM, Swift SE, Berghmans $\mathrm{B}$, Lee $\mathrm{J}$, et al. An international urogynecological association (IUGA)/international continence society (ICS) joint report on the terminology for female pelvic floor dysfunction. Neurourology and Urodynamics. 2010; 29: 4-20.

[4] Saboia DM, Firmiano MLV, Bezerra KDC, Vasconcelos JA, Oriá MOB, Vasconcelos CTM. Impact of urinary incontinence types on women's quality of life. Revista da Escola de Enfermagem da USP. 2017; 51: e03266.

[5] Krhut J, Gärtner M, Mokris J, Horcicka L, Svabik K, Zachoval $\mathrm{R}$, et al. Effect of severity of urinary incontinence on quality of life in women. Neurourology and Urodynamics. 2018; 37: 1925-1930.

[6] Duralde ER, Rowen TS. Urinary Incontinence and Associated Female Sexual Dysfunction. Sexual Medicine Reviews. 2017; 5: 470-485.

[7] Felippe MR, Zambon JP, Girotti ME, Burti JS, Hacad CR, Cadamuro L, et al. What is the Real Impact of Urinary Incontinence on Female Sexual Dysfunction? A Case Control Study. Sexual Medicine. 2017; 5: e54-e60.

[8] Brubaker L, Chiang S, Zyczynski H, Norton P, Kalinoski DL, Stoddard A, et al. The impact of stress incontinence surgery on female sexual function. American Journal of Obstetrics and Gynecology. 2009; 200: 562.e1-562.e7.

[9] Jha S, Strelley K, Radley S. Incontinence during intercourse: myths unravelled. International Urogynecology Journal. 2012; 23: 633-637.

[10] Salonia A, Zanni G, Nappi RE, Briganti A, Dehò F, Fabbri F, et al. Sexual dysfunction is common in women with lower urinary tract symptoms and urinary incontinence: results of a crosssectional study. European Urology. 2004; 45: 642-648. 
[11] Espuña Pons M, Puig Clota M. Coital urinary incontinence: impact on quality of life as measured by the King's Health Questionnaire. International Urogynecology Journal and Pelvic Floor Dysfunction. 2008; 19: 621-625.

[12] Nilsson M, Lalos O, Lindkvist H, Lalos A. Impact of female urinary incontinence and urgency on women's and their partners' sexual life. Neurourology and Urodynamics. 2011; 30: 12761280.

[13] Pinheiro Sobreira Bezerra LR, Britto DF, Ribeiro Frota IP, Lira do Nascimento S, Morais Brilhante AV, Lucena SV, et al. The Impact of Urinary Incontinence on Sexual Function: a Systematic Review. Sexual Medicine Reviews. 2020; 8: 393-402.

[14] Zahariou A, Karamouti M, Tyligada E, Papaioannou P. Sexual function in women with overactive bladder. Female Pelvic Medicine \& Reconstructive Surgery. 2010; 16: 31-36.

[15] Roos A, Thakar R, Sultan AH, Burger CW, Paulus ATG. Pelvic floor dysfunction: women's sexual concerns unraveled. The Journal of Sexual Medicine. 2014; 11: 743-752.

[16] Rogers RG, Pauls RN, Thakar R, Morin M, Kuhn A, Petri E, et al. An International Urogynecological Association (IUGA)/International Continence Society (ICS) joint report on the terminology for the assessment of sexual health of women with pelvic floor dysfunction. Neurourology and Urodynamics. 2018; 37: 1220-1240.

[17] Serati M, Salvatore S, Uccella S, Nappi RE, Bolis P. Female Urinary Incontinence during Intercourse: a Review on an Understudied Problem for Women's Sexuality. The Journal of Sexual Medicine. 2009; 6: 40-48.

[18] Illiano E, Mahfouz W, Giannitsas K, Kocjancic E, Vittorio B, Athanasopoulos A, et al. Coital Incontinence in Women with Urinary Incontinence: an International Study. The Journal of Sexual Medicine. 2018; 15: 1456-1462.

[19] Pastor Z. Female ejaculation orgasm vs. coital incontinence: a systematic review. The Journal of Sexual Medicine. 2013; 10: 1682-1691.

[20] Lau H, Huang W, Su T. Urinary leakage during sexual intercourse among women with incontinence: Incidence and risk factors. PLoS ONE. 2017; 12: e0177075.

[21] El-Azab AS, Yousef HA, Seifeldein GS. Coital incontinence: relation to detrusor overactivity and stress incontinence. Neurourology and Urodynamics. 2011; 30: 520-524.

[22] Pastor Z, Chmel R. Differential diagnostics of female "sexual" fluids: a narrative review. International Urogynecology Journal. 2018; 29: 621-629.

[23] Rodriguez FD, Camacho A, Bordes SJ, Gardner B, Levin RJ,
Tubbs RS. Female ejaculation: an update on anatomy, history, and controversies. Clinical Anatomy. 2021; 34: 103-107.

[24] Gray T, Li W, Campbell P, Jha S, Radley S. Evaluation of coital incontinence by electronic questionnaire: prevalence, associations and outcomes in women attending a urogynaecology clinic. International Urogynecology Journal. 2018; 29: 969-978.

[25] Grzybowska ME, Wydra DG. Coital incontinence: a factor for deteriorated health-related quality of life and sexual function in women with urodynamic stress urinary incontinence. International Urogynecology Journal. 2017; 28: 697-704.

[26] Martinez CS, Ferreira FV, Castro AAM, Gomide LB. Women with greater pelvic floor muscle strength have better sexual function. Acta Obstetricia et Gynecologica Scandinavica. 2014; 93: 497-502.

[27] Meyer S, Salchli F, Bettaieb H, Vial Y, Baud D, Fornage S, et al. The peripheral sexual response ... from urogynecology to sexology. Revue Medicale Suisse. 2015; 11: 2320-2325. (In French)

[28] Kanter G, Rogers RG, Pauls RN, Kammerer-Doak D, Thakar R. A strong pelvic floor is associated with higher rates of sexual activity in women with pelvic floor disorders. International Urogynecology Journal. 2015; 26: 991-996.

[29] Dumoulin C, Hay-Smith J. Pelvic floor muscle training versus no treatment, or inactive control treatments, for urinary incontinence in women. The Cochrane Database of Systematic Reviews. 2018; 10: CD005654.

[30] Bø K, Talseth T, Vinsnes A. Randomized controlled trial on the effect of pelvic floor muscle training on quality of life and sexual problems in genuine stress incontinent women. Acta Obstetricia et Gynecologica Scandinavica. 2000; 79: 598-603.

[31] Balzarro M, Rubilotta E, Mancini V, Trabacchin N, Oppezzi L, Li Marzi V, et al. Impact of Overactive Bladder-Wet Syndrome on Female Sexual Function: a Systematic Review and MetaAnalysis. Sexual Medicine Reviews. 2019; 7: 565-574.

[32] Wang Y, Shi C, Wang Y, Jiao W, Wang X, Zhang J, et al. The Correlation between Severity of Overactive Bladder Symptoms with Female Sexual Dysfunction and Sexual Satisfaction of Partners. Urologia Internationalis. 2021; 105: 124-130.

[33] Lai S, Diao T, Zhang W, Seery S, Zhang Z, Hu M, et al. Sexual Functions in Women with Stress Urinary Incontinence after Mid-Urethral Sling Surgery: a Systematic Review and MetaAnalysis of Prospective Randomized and Non-Randomized Studies. The Journal of Sexual Medicine. 2020; 17: 1956-1970.

[34] Atılgan AE, Eren EC. The effect of tension-free vaginal tape on coital incontinence concomitant with stress urinary incontinence. Lower Urinary Tract Symptoms. 2021; 13: 118-122. 